\title{
ATLAS TDAQ DataCollection Software
}

\author{
Christian Haeberli, Andre dos Anjos, Hans Peter Beck, Andre Bogaerts, David Boterill, Szymon \\ Gadomski, Piotr Golonka, Reiner Hauser, Emil Knezo, Micheal LeVine, Remigius Mommsen, Valeria \\ Perez Reale, Stefan Stancu, Jim Schlereth, Per Werner, Fred Wickens and Haimo Zobernig
}

On behalf of the ATLAS TDAQ DataFlow community [1]

\begin{abstract}
The DataCollection is a subsystem of the ATLAS Trigger and DAQ system. It is responsible for the movement of event data from the ReadOut subsystem to the Second Level Trigger and to the Event Filter. This functionality is distributed on several software applications running on Linux PCs interconnected with Gigabit Ethernet. For the design and implementation of these applications a common approach has been adopted. This approach leads to the design and implementation of a common DataCollection software framework providing a suite of common services.
\end{abstract}

\section{INTRODUCTION}

$T^{\mathrm{H}}$ HE Large Hardron Collider (LHC), which is currently under construction at CERN and will be operational in 2007, will collide $7 \mathrm{TeV}$ protons. ATLAS is a multi-purpose experiment dedicated to explore the discovery potential of the LHC.

Bunches of $10^{11}$ protons will collide at periods of $25 \mathrm{~ns}$ at the interaction point. This will result in $\sim 25$ proton-proton collisions per bunch crossing and a total interaction rate of $\sim 1 \mathrm{GHz}$. An online selection needs to be applied in order to select the 100 events per second with the highest discovery potential and to reduce the event rate by a factor of $10^{7}$. This

Manuscript received May 22, 2003.

Christian Haeberli, Hans Peter Beck and Valeria Perez Reale are with the Laboratory for High Energy Physics, University of Bern, 3012 Bern, Switzerland (telephone: $+41 \quad 31 \quad 631 \quad 40$ 64, e-mail: christian.haeberli@cern.ch).

Andre dos Anjos, Universidade Federal do Rio de Janeiro, COPPE/EE, Rio de Janeiro, Brazil

Andre Bogaerts, Piotr Golonka, Emil Knezo, Stefan Stancu and Per Werner are with CERN, 1211 Genève 23, Switzerland

Szymon Gadomski is with the Laboratory for High Energy Physics, University of Bern, Switzerland and with the Henryk Niewodniczanski Institute of Nuclear Physics, Cracow, Poland

Reiner Hauser is with the Michigan State University Department of Physics and Astronomy, East Lansing, Michigan, US

Micheal LeVine is with the Brookhaven National Laboratory (BNL), Upton, New York, US

Remigius Mommsen is with the University of California, Irvine, California, US

Jim Schlereth is with the Argonne National Laboratory, Argonne, Illinois, US

Fred Wickens and David Boterill are with the Rutherford Appleton Laboratory, Chilton, Didcot, UK

Haimo Zobernig is with the Department of Physics, University of Wisconsin, Madison, Wisconsin reduction allows to store only a manageable and analyzable amount of data.

ATLAS wants to achieve the online event selection with a three level trigger system. The first level trigger is custom build hardware, whereas the High Level Triggers (HLT), which comprises the second level trigger (LVL2) and the Event Filter, are software algorithms running on commodity PCs..

The Data-flow system is responsible for the readout of detector data, the serving of data to the High Level Triggers system (HLT). The Data-flow system also moves data accepted by the HLT to mass storage. The ReadOut subsystem (ROS) groups multiple ReadOut links from the detector, buffers the incoming data and makes it available for the DataCollection subsystem which transports the data to the LVL2 and the Event Filter. A more detailed description of the Data-flow system is given in [2].

The DataCollection (DC) subsystem transports regions-ofinterest (RoI) data, as identified by the first level trigger system (LVL1), from the ROS to the LVL2 and full events to the Event Filter. In addition it transports event data from the Event Filter to mass storage.

The basic requirements for the DataCollection subsystem are to transport RoIs of an average size of $16 \mathrm{kB}$ at the LVL1 accept rate of $100 \mathrm{kHz}$ to the LVL2 processors and to build full sized events of $1.2 \mathrm{MB}$ at a LVL2 accept rate of $\sim 3 \mathrm{kHz}$ for the LHC startup luminosity. Therefore the bandwidth into Level 2 is $1.6 \mathrm{~GB} / \mathrm{s}$ and the bandwidth into the Event Filter is $3.6 \mathrm{~GB} / \mathrm{s}$.

The functionality of DataCollection is distributed to six software applications running on Linux PCs interconnected with Gigabit Ethernet. A common approach to design and implementation was chosen, which leads to the design and implementation of a common DataCollection software framework, providing a suite of common services (e.g. Message Passing and Application control). The design and the implementation of the DC framework are based on $\mathrm{C}++$ and the standard template library (STL). The DC applications are multithreaded and built on top of the framework.

\section{DistRibution OF FUnCTIONALITY}

The DataCollection functionality is distributed to six software applications: the LVL2 Supervisor (L2SV), the 
LVL2 processing unit (L2PU), the pseudo ROS (pROS), the Data-flow manager (DFM), the Subfarm-input (SFI) and the Subfarm-output (SFO). An overview of the DC applications and their interactions is shown in Fig. 1.

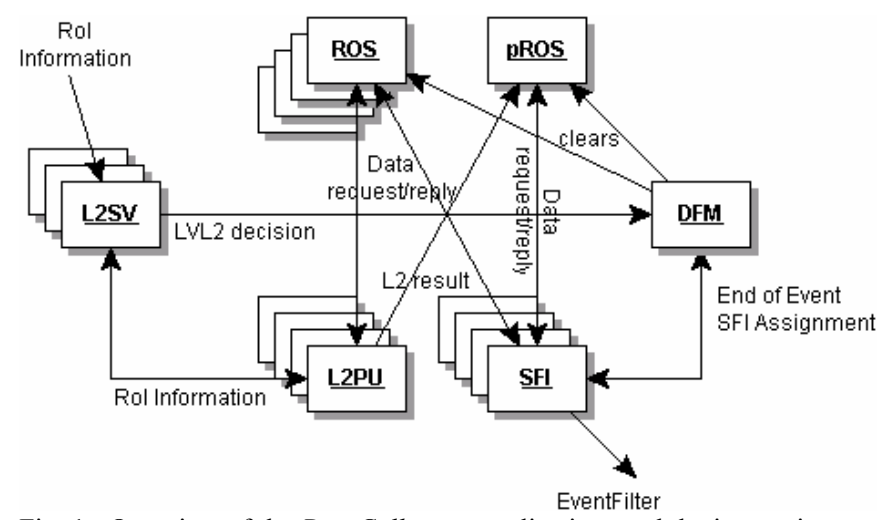

Fig. 1. Overview of the DataCollection applications and the interactions inbetween them.

\section{A. LVL2 Supervisor}

The L2SV receives a LVL1 decision containing geometry information of RoIs from the region-of-interest builder. It load balances a part of the LVL2 farm consisting of many L2PUs while assigning events to L2PUs chosen according to a load-balancing algorithm. After processing at the L2PU the L2SV receives the LVL2 decisions, which it forwards to the DFM.

\section{B. LVL2 Processing Unit}

The L2PU receives a LVL1 decision from the L2SV. It requests the RoI data it needs for processing from many ROSs. After the LVL2 decision is taken it reports it to the $L 2 S V$. In addition it sends a more detailed LVL2 result to the pROS.

The $D C$ framework provides the basic services needed by the LVL2 algorithms. The decision taking LVL2 algorithms are beyond the scope of the DC software, but it builds the basis for them.

\section{Pseudo ROS}

The pROS is the interface between the LVL2 and the Event Filter. It receives detailed LVL2 results from the L2PUs and takes part in the event building as a common ROS. It ensures that the detailed LVL2 result becomes a part of the fully built event and therefore is accessible for the algorithms running in the Event Filter.

\section{Data-flow Manager}

The DFM is responsible for the load balancing and the bookkeeping for the event building. It receives the $L V L 2$ decisions from the L2SV and forwards the LVL2 rejects to all instances of the ROS to ensure the deletion of these events. For every LVL2 accept the DFM assigns an SFI for event building.

\section{E. Subfarm-input}

The SFI is fulfilling the major part of the event building functionality. It gets an event assigned by the DFM and requests the event fragments from all instances of the ROS. As soon as all fragments of a certain event arrived at the SFI an end-of-event message is sent to the DFM. The fully built event is kept and made accessible to the Event Filter.

\section{F. Subfarm-output}

The SFO receives events accepted by the Event Filter. It buffers these in files held on a local disk and makes the files available to the mass storage system.

\section{FRAMEWORK FUNCTIONALITY}

The framework provides a suite of common functionalities to the DataCollection applications. These services include MessagePassing, Application Control, Error Reporting, Configuration Database, System Monitoring, OS Abstraction Layer, Event Formatting and Time Stamping. A part of these framework packages (e.g. Application control) need to interface to the common TDAQ control software services, provided by the OnlineSW suite [3]. An architectural view of the DataCollection framework is shown in Fig. 2.

\begin{tabular}{|c|c|c|c|c|c|c|c|}
\hline \multicolumn{8}{|c|}{ DC Application Code } \\
\hline \multicolumn{3}{|c|}{ Message Passing } & \multirow{2}{*}{ 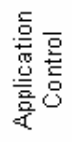 } & \multirow{2}{*}{ 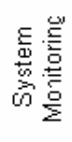 } & \multirow{2}{*}{ 高 } & \multirow{2}{*}{ 䓌 } & \multirow{2}{*}{ 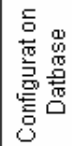 } \\
\hline $\mathrm{TCP}$ & UDP & $\begin{array}{c}\text { raw } \\
\text { Ethernet }\end{array}$ & & & & & \\
\hline \multicolumn{8}{|c|}{ OS Layer } \\
\hline
\end{tabular}

Fig. 2. Architectural view of the DataCollection framework.

\section{A. Message passing}

The Message passing layer is responsible for the transfer of event data and control messages between Data-flow components. The latter ensure the proper movement of the data. It imposes no structure on the data, which is to be exchanged, except a four-byte alignment of the byte-stream. It allows the transfer of data blocks of up to $64 \mathrm{kB}$ size with a best-effort guarantee. For efficiency reasons this layer does no re-transmission or acknowledgment of data. This choice has allowed the API to be implemented over a range of technologies without imposing an un-necessary overhead or the duplication of existing functionality, e.g. in the case of TCP/IP. The API supports the sending of both unicast and multicast messages. The latter may be emulated to the $D C$ application code in case the underlying protocol does not support multicast, e.g. for TCP/IP.

The design of the Message Passing layer defines classes that allow the sending and receiving of messages. The Node, Group and Address classes are used at configuration time to setup all the necessary internal connections. The Port class is the central interface for sending data. All user data has to be 
part of a Buffer object to enable it to be sent or received from a Port. The Buffer interface allows for addition of user defined memory locations without copying. The Provider class is an internal interface from which different protocol and technology specific implementations inherit. Multiple Provider objects can be active at any given time allowing the concurrent use of different protocols. To date providers implementing TCP/IP, UDP/IP and raw Ethernet exist. The design is open for more protocols to be added in the future.

As the Message passing layer itself does not guarantee the reliable delivery of messages via the network (except if running a reliable underlying network protocol) the DC application code has to implement strategies to avoid message loss and to recover from packet loss.

We have mainly observed message loss in input buffers of switches and in the Linux kernel input buffer, despite the kernel buffer size was increased to $8 \mathrm{MB}$. A large fraction of this loss can be avoided by limiting the number of outstanding requests in a request-reply traffic pattern. This measure avoids any packet loss in the kernel input buffer because one can determine the maximum total size of buffered messages. In addition it decreases the probability for packet loss in the input buffers of the switches because one can determine the maximum number of messages travelling through the network at any time.

As limiting the number of outstanding requests can minimize the packet loss but not exclude it absolutely, some DC applications implement a re-ask mechanism for missing messages in request-reply traffic.

\section{B. Application Control}

The run control interface is responsible for translating state transition commands issued by a run controller application into commands internal to the DC application. The two applications communicate via a dedicated TCP/IP stream. Alternatively a keyboard interface has been defined, allowing for running DC applications in a local environment. The application control class is instantiated in the DC applications and provides virtual methods for all state transition to be implemented by the application developer.

\section{Error Reporting}

The Error Reporting package allows the logging of error messages either to standard output and standard error or to message reporting service (MRS) provided by the OnlineSW. Each DC software package can define its own set of error messages and error codes. Error logging can be enabled/disabled on a per package basis. Furthermore debug messages and error messages are treated logically differently, so the debug message could go to standard output while all normal application logs go to MRS. The user only interfaces via a set of macros to the ErrorReporting system allowing optimization of the applications at compile time.

\section{Configuration Database}

All DC applications obtain their configuration parameters from the configuration database provided by the Online $S W$.
The access layer to the configuration database allows the underlying implementation to change without implying changes to the application. The application's view of the database is hidden by configuration objects, which access the database, providing a more convenient way to access configuration information. The configuration objects themselves are created by a code generator, which parses the configuration database schema file.

\section{E. System Monitoring}

This part of the framework allows every DC application to make arbitrary information available to some outside client. In practice this is used to publish statistics like counters or rates. The package makes this information available in various different ways, including the Online $S W$.

\section{F. OS Abstraction Layer}

The OS abstraction layer consists of packages hiding all OS specific interfaces. E.g. the threads package hides the details of the underlying POSIX thread interface, which could in the future be replaced by another implementation

\section{G. Event Format}

This package allows to format event data according to [4], to navigate through this structure and to store event data in dedicated storage types. One important example is the $\mathrm{I} / \mathrm{O}$ vector storage type, which holds a vector of pointers to event fragments. This storage type is used to avoid copying if data fragments of a specific event are distributed to many places in memory and thus allows for scatter-gather send operations avoiding memory copies when shipping out data.

\section{H. Time Stamping}

The Time stamping library provides hooks to resolve the timing behavior of the software. Two dedicated implementations exist. The first one is based on NetLogger [5] and used to understand the timing behavior of a distributed system; the second provides fine-grained time resolution to understand the timing behavior inside a single application. The output of both implementations satisfies the NetLogger format and therefore the NetLogger visualization tool [5] can be used to analyze the data generated by both of them.

\section{PERFormance ReQUiREMENTS FOR DATACOLlECTION} APPLICATIONS

TABLE I

MESSAGE RATES AND BANDWIDTHS OF CONTROL AND DATA MESSAGES BETWEEN THE DATACOLLECTION COMPONENTS AN THE ROS 


\begin{tabular}{|c|c|c|c|c|c|c|}
\hline & \multirow{2}{*}{\multicolumn{2}{|c|}{$\begin{array}{l}\text { Message } \\
\text { Type }\end{array}$}} & \multicolumn{2}{|c|}{ Sender } & \multicolumn{2}{|c|}{ Receiver } \\
\hline & & & Rate & Bandwidth & Rate & Bandwidth \\
\hline $\mathrm{L} 2 \mathrm{SV} \rightarrow \mathrm{L} 2 \mathrm{PU}$ & Rol information & & $7.5 \mathrm{kHz}$ & $5 \mathrm{MB} / \mathrm{s}$ & $0.5 \mathrm{kHz}$ & $0.3 \mathrm{MB} / \mathrm{s}$ \\
\hline \multirow{2}{*}{$\mathrm{L} 2 \mathrm{PU} \rightarrow \mathrm{ROS}$} & \multirow{2}{*}{ Data requests } & b) & $6 \mathrm{kHz}$ & $0.6 \mathrm{MB} / \mathrm{s}$ & $16 \mathrm{kHz}$ & $1.6 \mathrm{MB} / \mathrm{s}$ \\
\hline & & s) & $1 \mathrm{I} \mathrm{kHz}$ & $1 \mathrm{MB} / \mathrm{s}$ & $6 \mathrm{kHz}$ & $0.6 \mathrm{MB} / \mathrm{s}$ \\
\hline \multirow{2}{*}{$\mathrm{ROS} \rightarrow \mathrm{L} 2 \mathrm{PU}$} & \multirow{2}{*}{ Event data } & b) & $16 \mathrm{kHz}$ & $32 \mathrm{MB} / \mathrm{s}$ & $6 \mathrm{kHz}$ & $11 \mathrm{MB} / \mathrm{s}$ \\
\hline & & s) & $6 \mathrm{kHz}$ & $6 \mathrm{MB} / \mathrm{s}$ & $11 \mathrm{kHz}$ & $11 \mathrm{MB} / \mathrm{s}$ \\
\hline $\mathrm{L} 2 \mathrm{PU} \rightarrow \mathrm{L} 2 \mathrm{SV}$ & \multicolumn{2}{|l|}{ LVL2 decisions } & $0.5 \mathrm{kHz}$ & $50 \mathrm{kB} / \mathrm{s}$ & $7.5 \mathrm{kHz}$ & $750 \mathrm{kB} / \mathrm{s}$ \\
\hline $\mathrm{L} 2 \mathrm{SV} \rightarrow \mathrm{DFM}$ & \multicolumn{2}{|l|}{ LVL2 decision ${ }^{z)}$} & $75 \mathrm{~Hz}$ & $40 \mathrm{kB} / \mathrm{s}$ & $750 \mathrm{~Hz}$ & $400 \mathrm{kB} / \mathrm{s}$ \\
\hline $\mathrm{DFM} \rightarrow \mathrm{SFI}$ & Assign event & & $3 \mathrm{kHz}$ & $0.3 \mathrm{MB} / \mathrm{s}$ & $30 \mathrm{~Hz}$ & $3 \mathrm{kB} / \mathrm{s}$ \\
\hline \multirow{2}{*}{$\mathrm{SFI} \rightarrow \mathrm{ROS}$} & \multirow{2}{*}{ Data request } & b) & $4 \mathrm{kHz}$ & $0.4 \mathrm{MB} / \mathrm{s}$ & $3 \mathrm{kHz}$ & $0.3 \mathrm{MB} / \mathrm{s}$ \\
\hline & & s) & $48 \mathrm{kHz}$ & $4.8 \mathrm{MB} / \mathrm{s}$ & $3 \mathrm{kHz}$ & $0.3 \mathrm{MB} / \mathrm{s}$ \\
\hline \multirow{2}{*}{$\mathrm{ROS} \rightarrow \mathrm{SFl}$} & \multirow{2}{*}{ Event data } & b) & $3 \mathrm{kHz}$ & $36 \mathrm{MB} / \mathrm{s}$ & $4 \mathrm{kHz}$ & $48 \mathrm{MB} / \mathrm{s}$ \\
\hline & & s) & $3 \mathrm{kHz}$ & $3 \mathrm{MB} / \mathrm{s}$ & $48 \mathrm{kHz}$ & $48 \mathrm{MB} / \mathrm{s}$ \\
\hline $\mathrm{SFI} \rightarrow \mathrm{DFM}$ & Finished Event & & $30 \mathrm{~Hz}$ & $3 \mathrm{kB} / \mathrm{s}$ & $3 \mathrm{kHz}$ & $0.3 \mathrm{MB} / \mathrm{s}$ \\
\hline $\mathrm{DFM} \rightarrow \mathrm{ROS}$ & Clear buffers ${ }^{g}$ & & $250 \mathrm{~Hz}$ & $0.4 \mathrm{MB} / \mathrm{s}$ & $250 \mathrm{~Hz}$ & $0.4 \mathrm{MB} / \mathrm{s}$ \\
\hline
\end{tabular}

There are two kinds of I/O performance requirements for the DataCollection subsystem: message rates and bandwidth. Table I summarizes rate and bandwidth requirements for the different transactions between the DataCollection applications and the ROS. The SFI has to fulfill the most demanding $\mathrm{I} / \mathrm{O}$ requirements of all the applications.

\section{DataCollection Framework Performance}

The performance of the DataCollection software was validated in an extensive measurements program. Its detailed results are presented in [6].

\section{A. Aim of the Performance Tests}

A DataCollection application will not use all framework services when running, but rather at earlier states e.g. configuration. Another part of the framework functionality is used in the running state, but at a low rate only (e.g. error reporting and system monitoring). These parts of the framework are not critical for the performance of the running system. In a stably running setup error messages are occurring very rarely only (less than one per second). The monitoring service is running at a rate of $\sim 1 \mathrm{~Hz}$ in a separate thread. This thread is flushing counter values which are continuously updated by the performance critical working threads to the monitoring service of the Online Software. All measurements provided with the DataCollection software were obtained with the error reporting and system monitoring facilities enabled. The measurements even rely on the system monitoring as the service provides the information which is needed to obtain the results of the measurements.

The framework functionalities, which are used at a high rate in the running state, are the message passing, the event formatting and the OS services. Therefore measuring the performance of a DC application implies also to validate the framework performance. To achieve this validation it is sufficient to demonstrate that the DC application with the most demanding $\mathrm{I} / \mathrm{O}$ performance meets its requirements. Therefore a measurement using the SFI, which is the DC application with the most demanding I/O performance (see Table I), is the validation of the DC framework performance and is presented in this paper.

\section{B. Setup of the Permance Tests}

The tests were provided on the Dataflow performance testbed which is described in [2]. The SFI application was running on a dual Intel Xeon $2.4 \mathrm{GHz}$ CPU PC with $1 \mathrm{~GB}$ RAM. The operating system was the CERN certified version of Redhat Linux 7.2.

The SFI was interconnected to 60 FPGA ROS emulators via two layers of Ethernet Switches: the first layer switch was a 31 ports Gigabit Ethernet BATM T6 which connected the SFIs to two concentrator switches. The concentrator switches were 32 ports Fast Ethernet BATM T5 with two Gigabit Ethernet uplink connections. These connected each 30 FPGA ROS emulators to the central switch using one of the uplinks. The measurement in Fig. 3. shows the input bandwidth of the SFI performing event building from 60 FPGA ROS emulators [7] simulating 1600 data sources.

The SFI was operating in pull mode. It was sending 1600 unicast requests in a round robin pattern to the FPGA ROS emulators. Raw Ethernet was applied as network protocol, because it is implemented in the FPGA ROS Emulators exclusively.

\section{Results and Discussion}

The plot in Fig. 3 shows that the SFI total message rate depends very little on the fragment size $(131 \mathrm{kHz}$ message rate for 160 Byte fragments vs. $114 \mathrm{kHz}$ for 1456 Byte fragments). The SFI application is running in the CPU limited domain and it is exploiting two third of the Gigabit Ethernet bandwidth. As the SFI performance is purely CPU limited the bandwidth the SFI can drive is expected to scale linearly with the CPU speed of the SFI machine. Therefore it is expected that the application will be able to drive the full Gigabit Ethernet bandwidth on a $3.6 \mathrm{GHz}$ CPU platform, which will become available well before the startup of LHC. In addition we expect performance gains from further optimizations of the DC code. The SFI is building events exclusively and is not processing any event data. Therefore it is not critical to use the full or a large fraction of its CPU capacity for data movement. The SFI meets the message rate requirement of $96 \mathrm{kHz}$ and the bandwidth requirement of $48 \mathrm{MB} / \mathrm{s}$.

It is straightforward to translate the SFI performance results to other applications e.g. the L2PU. Given the fact that the SFI needs almost all its CPU resources to drive 80 $\mathrm{MB} / \mathrm{s}$ input bandwidth, the L2PU will need $15 \%$ of its CPU resources for $\mathrm{I} / \mathrm{O}$, the remaining resources will be available for processing the decision taking algorithms for LVL2. This estimation is pessimistic, as in opposite to the SFI the L2PU does not need to do any event building operations. Measurements with the L2PU are indicating a worst case value of $10 \%$ [8]. 
All the other DC applications have much lower rate and bandwidth requirements than the SFI (see Table I). Therefore the performance of the DC framework suits the needs of these applications.

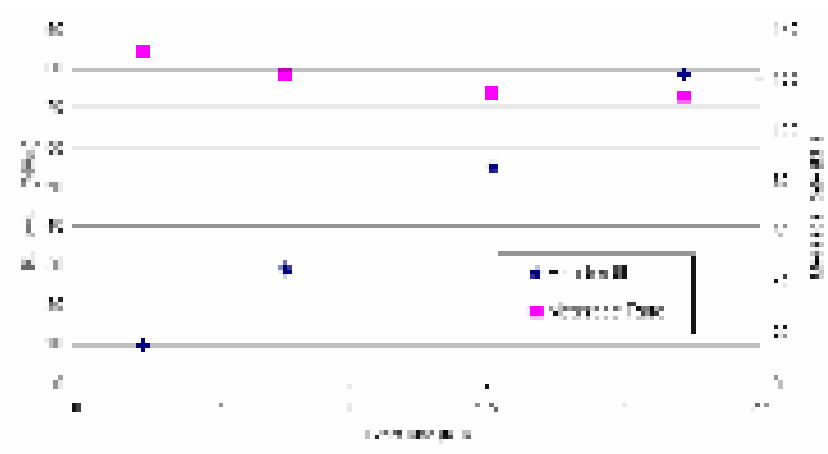

Fig. 3. The SFI bandwidth and message rate running against 60 FPGA ROS emulators acting as 1600 data sources. The fragment size per data source was varied from 160 to 1456 Bytes. Adding the header information of the DC raw Ethernet implementation this fragment size is a full Ethernet frame of 1500 Bytes. The variation of the fragment size means that the event size was varied from 0.2 to $2.2 \mathrm{MB}$.

\section{Network Protocols}

As for most of the DC performance measurements ROS emulators were used [7] and as those have only the raw socket protocol implemented, the DC group is mainly experienced with this protocol. We expect the UDP/IP protocol to behave similarly as raw Ethernet, as both are connectionless protocols. Measurements showed that the raw Ethernet protocol was faster for single frame messages than UDP/IP [9]. For multi-frame messages the opposite applies.

The application of TCP/IP for RoI collection and event building is not appropriate due to the following reasons [10]:

1) TCP/IP is a connection oriented protocol, every application has to hold a connection to each partner application it communicates to. E.g. the SFI needed to hold up to 1600 connections. This may lead to a scalability problem

2) The sliding windows mechanism is not appropriate for event building. If a package gets lost, no data can be exchanged between two communication partners. This means that no other event can be completely built before the TCP recovery mechanism took place and the lost package arrives at the SFI. This leads to a latency problem.

3) $\mathrm{TCP} / \mathrm{IP}$ is a reliable protocol. In order to achieve the reliability the protocol issues acknowledgment messages to confirm the reception of a data packet. Depending on the round trip time of the network, TCP/IP can increase the message rate in the network up to $50 \%$ due two acknowledgement messages. This takes network and CPU resources.

4) Long term tests, running event building with a connectionless protocol showed a rate of package loss of $10^{-9}$. This minimal loss is caught by a recovery mechanism on the level of the SFI application. This means that there is no need for a reliable protocol and its potential drawbacks.

Therefore TCP/IP will not be used for data traffic (event building and RoI collection). However the protocol could be used for control messages like Level 2 decisions or End of Event notifications.

\section{CONCLUSIONS}

Thanks to the framework approach it was possible to develop the DC software within two years. In addition this approach will ease future maintenance of the subsystem.

The performance of the applications built on top of the framework is promising and sufficient for ATLAS even with today's hardware. E.g. a DataCollection application running on a $2.4 \mathrm{GHz}$ dual CPU machine, can handle a total message rate of $114 \mathrm{kHz}$ in a request-reply traffic pattern (sending small request messages, receiving event data). Therefore the choice of the framework approach, Linux, C++, STL and multithreading was proved to be viable.

\section{ACKNOWLEDGMENT}

Christian Haeberli thanks Marian Zurek for the system administration of the testbed at CERN, which was the key facility to evaluate and improve the performance of the $D C$ software.

\section{REFERENCES}

[1] The ATLAS TDAQ DataFlow community. [Online]. Available: http://atlas.web.cern.ch/Atlas/GROUPS/DAQTRIG/DataFlow/DFlowA uthors.pdf ${ }^{1}$

[2] HP. Beck, M. Abolins, A. Dos Anjos, M. Barisonzi, M. Beretta, R. Blaier et al., "The base line DataFlow system of the ATLAS Trigger \& DAQ," in Proc. $13^{\text {th }}$ IEEE NPSS Real Time Conferene, 2003

[3] I. Alexandrov, A. Amorin, E. Badescu, M. Barczyk, D. BurckhartChromek, M. Caprini et al., "Online software for the ATLAS test beam data acquisition system," in Proc. $13^{\text {th }}$ IEEE NPSS Real Time Conferene, 2003

[4] C. Bee, D. Francis, L. Mapelli, R. McLaren, G. Mornacchi, J. Petersen et al., "The raw event format in the ATLAS Trigger \& DAQ, ATLAS Internal Note ATL-DAQ-98-129, Oct. 2002

[5] B. Tierney and D. Gunter, "NetLogger: a toolkit for distributed system performance tuning and debugging", LBNL Tech Report LBNL-51276, Dec. 2002

[6] The ATLAS TDAQ community, "ATLAS high level trigger, data acquisition and controls", CERN / LHCC / 2003-022

[7] M. LeVine, S. Stancu, Ch. Haeberli, L. Tremblet, R. Beuran, C. Meirosu et al., "Network performance investigation for the ATLAS Trigger/DAQ", in Proc. 13 $3^{\text {th }}$ IEEE NPSS Real Time Conferene, 2003

[8] M. Abolins, S. Armstrong, J.T. Baines, M. Barisonzi, H.P. Beck, C.P. Bee et al., "The Second Level Trigger of the ATLAS experiment at

${ }^{1}$ M. Abolins, A. Dos Anjos, M. Barisonzi,, H.P. Beck, M. Beretta, R. Blair, J. Bogaerts, H. Boterenbrood, D. Botterill, M. Ciobotaru, E. Palencia Cortezon, R. Cranfield, G. Crone, J. Dawson, B. DiGirolamo, R. Dobinson, Y. Ermoline, M.L. Ferrer, D. Francis, S. Gadomski, S. Gameiro, P. Golonka, B. Gorini, B. Green, M. Gruwe, C. Haeberli, Y. Hasegawa, R. Hauser, C. Hinkelbein, R. Hughes-Jones, P. Jansweijer, M. Joos, A. Kaczmarska, G. Kieft, E. Knezo, K. Korcyl, A. Kugel, A. Lankford, G. Lehmann, M. LeVine, W. Liu, T. Maeno, M. Losada Maia, L. Mapelli, B. Martin, R. McLaren, C. Meirosu, A. Misiejuk, R. Mommsen, G. Mornacchi, M. Müller, Y. Nagasaka, K. Nakayoshi, I. Papadopoulos, V. Perez-Reale, J Petersen, P. de Matos Lopes Pinto, D. Prigent, J. Schlereth, M. Shimojima, R. Spiwoks, S. Stancu, J. Strong, L. Tremblet, J. Vermeulen, P. Werner, F. Wickens, Y. Yasu, M. Yu, H. Zobernig, M. Zurek 
CERN's LHC," presented at the Nuclear Sciences Symposium 2003, Portland, OR, USA, 2003

[9] Piotr Golonka, "Linux network performance study for the ATLAS data flow system". [Online]. Available: https://edms.cern.ch/document/368844

[10] Richard Hughes-Jones, "The use of TCP/IP for real-time messages in ATLAS Trigger/DAQ". [Online]. Available: https://edms.cern.ch/document/393752 